\title{
CARGA PARA A APLICAÇÃO DE TESTES DE I-RM EM EXERCÍCIOS DE MEMBROS SUPERIORES EM MULHERES JOVENS TREINADAS E NÃO TREINADAS'
}

\author{
MS. THIAGO MATTOS FROTA DE SOUZA \\ Curso de Graduação em Educação Física, Faculdades Integradas \\ Einstein de Limeira (Limeira - São Paulo - Brasil) \\ E-mail: thiago_mfs@hotmail.com
}

\section{GRAD. MÁRCIO ANTONIO GONSALVES SINDORF}

Faculdade de Ciências da Saúde, Universidade Metodista de Piracicaba (Piracicaba - São Paulo - Brasil)

E-mail: maasindorf@unimep.br

\section{MS. PAMELA ROBERTA GOMES GONELLI \\ Faculdade de Ciências da Saúde, Universidade Metodista de Piracicaba - UNIMEP (Piracicaba - São Paulo - Brasil) \\ E-mail: pargonel@unimep.br}

\section{MS. RICARDO ADAMOLI SIMÕES}

Programa de Pós-Graduação em Educação Física ,Universidade

Metodista de Piracicaba (Piracicaba - São Paulo - Brasil)

E-mail: aspone@hotmail.com

\section{DRA. MARIA IMACULADA DE LIMA MONTEBELO \\ Faculdade de Ciências Matemáticas e da Natureza, Universidade \\ Metodista de Piracicaba (Piracicaba - São Paulo - Brasil) \\ E-mail: milmonte50@gmail.com}

\section{DR. MARCELO DE CASTRO CESAR}

Faculdade de Ciências da Saúde, Universidade Metodista de Piracicaba (Piracicaba - São Paulo - Brasil)

E-mail: maccesar@unimep.br

I. Os autores agradecem à Fundação de Amparo à Pesquisa do Estado de São Paulo - FAPESP, pelo suporte financeiro e pelas bolsas de treinamento técnico, à Coordenação de Aperfeiçoamento de Pessoal de Nível Superior - CAPES, pelas bolsas de mestrado e ao Conselho Nacional de Desenvolvimento Científico e Tecnológico - CNPq, pelas bolsas de iniciação científica. 


\section{RESUMO}

O objetivo do estudo foi propor valores de referência para a aplicação dos testes de I-RM em mulheres jovens treinadas e não treinadas. 90 mulheres, de 18 a 31 anos, agrupadas em: 43 Treinadas (GT) e 47 Não Treinadas (GNT) foram submetidas aos testes de I-RM nos seguintes exercícios: supino reto, desenvolvimento, rosca direta e tríceps testa. O GT apresentou maiores valores em relação ao GNT, em termos absolutos e relativos: supino reto $(G T=36,44 \mathrm{~kg} / 0,63$ e $G N T=27,34 \mathrm{~kg} / 0,46)$, desenvolvimento $(G T=27,93 \mathrm{~kg} / 0,49$ e $G N T=22,70 \mathrm{~kg} / 0,38)$, rosca direta $(G T=21,63 \mathrm{~kg} / 0,37$ e $\mathrm{GNT}=15,57 \mathrm{~kg} / 0,26)$ e tríceps testa $(G T=18,09 \mathrm{~kg} / 0,31$ e $G N T=12,55 \mathrm{~kg} / 0,21)$. Os valores encontrados no estudo podem ser utilizados como referências de cargas para a aplicação dos testes de I-RM.

PALAVRAS-CHAVE: Educação fisica e treinamento; avaliação física; mulheres; força muscular.

\section{INTRODUÇÃO}

treinamento de força é utilizado para melhorar o rendimento em esportes, o condicionamento físico, a estética e a saúde (MCCARTNEY et al., I988; MCCARTNEY; MCKELVIE, 1996; BARBOSA et al., 2000; KRAEMER; RATAMESS, 2004) e a participação de mulheres em programas de treinamento com pesos é cada vez maior (MAYHEW et al., 2008).

Para a otimização da prescrição dos exercícios com pesos, a avaliação física é fundamental, pois esta é extremamente complexa devido a diferentes respostas de um mesmo indivíduo em condições distintas ou entre os indivíduos, quando submetidos ao mesmo estímulo (NAHAS, 200 I), devendo ser o treinamento sempre supervisionado por um profissional qualificado, para a prevenção de lesões e maximização dos resultados esperados (MAZZETTI et al., 2000; KRAEMER; RATAMESS, 2004).

Com relação à avaliação física no treinamento de força, diversos são os métodos propostos para avaliar a força muscular, incluindo testes indiretos por meio de equações ou submáximos (KRAVITZ et al., 2003; MAYHEW et al., 2008; DESGORCES et al., 20I0; RONTU et al., 20I0), garantindo assim uma maior segurança do avaliado ao não submetê-lo ao esforço máximo. Mas o teste de uma repetição máxima ( I-RM) é uma metodologia já consagrada e segura para avaliação da força muscular e, por meio de seus percentuais, posterior prescrição do treinamento de força para os mais diversos objetivos, sendo utilizado para comprovar a efetividade de um determinado protocolo de treinamento (MCCURDY et al., 2008; SOUZA et al., 2008; BRECHUE; MAYHEW, 2009; CESAR et al., 2009; BRECHUE; MAYHEW, 20I2).

Embora o teste de I-RM seja comprovadamente prático, de baixo custo operacional e metodologia segura de avaliação para a maioria das populações (DIAS et al., 2005), um dos cuidados para sua melhor aplicação, além da correta execução 
dos movimentos, é a determinação da carga inicial, auxiliando ou comprometendo a verificação da carga máxima dentro do número ideal de tentativas indicadas, além de poder expor o avaliado a uma carga demasiadamente superestimada, o que poderia levá-lo a uma possível lesão.

Apesar de muitas pesquisas utilizarem o teste de I-RM como método de avaliação da força muscular em mulheres (BARBOSA et al., 2000; ROTH; MARTEL; IVEY, 2000; POEHLMAN et al., 2002; DIAS et al., 2005b; WESTPHAL; BAPTISTA; OLIVEIRA, 2006; MATERKO; NEVES; SANTOS, 2007; BRENTANO et al., 2008; MAYHEW et al., 2008; SOUZA et al., 2008; CESAR et al., 2009), ainda são poucos os trabalhos que apresentam valores de referência para o teste de I-RM, principalmente com mulheres, havendo a necessidade de estudos que estabeleçam parâmetros para sua aplicação.

Sendo assim, o objetivo do presente estudo foi comparar a força muscular e propor valores de referência de cargas iniciais para a aplicação dos testes de I-RM para membros superiores em mulheres jovens treinadas e não treinadas.

\section{MÉTODOS}

\section{SUJEITOS}

Participaram do estudo 90 mulheres, idade entre 18 e 3 I anos, saudáveis, estudantes da Universidade Metodista de Piracicaba, que foram recrutadas de forma aleatória e agrupadas em dois grupos:

- Grupo I-Treinadas (GT): 43 mulheres, idade de 22,47 \pm 3,27 anos, participantes regulares de programas de treinamento com pesos há pelo menos três meses.

- Grupo II - Não Treinadas (GNT): 47 mulheres, idade de 20,74 + 3,14 anos, que não participavam de qualquer programa regular de treinamento físico há pelo menos três meses e não estavam envolvidas em nenhum tipo de treinamento regular durante a realização do estudo.

Após a explicação da pesquisa, as voluntárias assinaram o termo de consentimento livre e esclarecido. $O$ presente estudo é parte integrante de um projeto-temático aprovado pelo Comitê de Ética em Pesquisa da Universidade Metodista de Piracicaba (Protocolo $n^{\circ}$ 83/03). As participantes também foram submetidas a uma avaliação clínica inicial composta por exame físico e anamnese, conduzida por um médico antes do início do estudo, para descartar qualquer contraindicação aos testes de I-RM, determinando se as voluntárias estavam aptas ou não a participarem do estudo.

Como critérios de exclusão, foram adotados os seguintes: o uso de medicamentos, apresentarem doenças cardíacas, ortopédicas, asma, hipertensão arterial e estarem engajadas em qualquer programa de treinamento. 
Foram realizadas as seguintes medidas antropométricas:

- estatura: utilizando o estadiômetro Alturaexata® com precisão de $0,1 \mathrm{~cm}$, onde a voluntária permanecia na posição ortostática sobre a plataforma, com os pés unidos e de costas para o equipamento, realizando uma inspiração profunda para a aferição da estatura, verificando a distância entre a região plantar e o vértex.

- massa corporal: utilizando a balança mecânica Welmy ${ }^{\circledR}$ devidamente calibrada e com precisão de 100g, onde a voluntária permanecia imóvel na posição ortostática sobre o centro da plataforma.

Em seguida foi calculado o Índice de Massa Corporal (IMC) pela massa corporal/estatura. ${ }^{2}$

\section{TESTES DE UMA REPETIÇÃO MÁXIMA (I-RM)}

Para determinação da força muscular, foram realizados testes de I-RM. Os testes eram realizados na mesma ordem (supino, desenvolvimento, rosca e tríceps) e sempre conduzidos pelos mesmos avaliadores, que eram treinados para tal procedimento, procedendo da seguinte maneira: ao realizar uma repetição correta, cargas extras eram acrescentadas até o comprometimento da execução do exercício. Nesse caso, uma nova tentativa era realizada com um peso intermediário entre o último levantamento correto e a repetição da falha, determinando a I-RM no nível desejado de precisão, que deveria ser encontrada em até cinco tentativas, com intervalos de três minutos entre elas. Esta técnica foi usada com barras, halteres e anilhas (BROWN; WEIR, 200 I). Antes da aplicação dos testes de I-RM, as voluntárias realizavam um aquecimento e alongamento com exercícios balísticos (três exercícios para membros superiores, 20 movimentos ativos cada exercício) e 10 repetições com uma carga leve (carga subjetiva e confortável utilizada nas sessões de familiarização) no exercício: flexão horizontal de ombros e extensão de cotovelos no supino reto, para prevenir possíveis lesões (MCCARTHY et al., 1995; WILBER et al., 1995; ROTH; MARTEL; IVEY, 2000; HURLBUT et al., 2002; POEHLMAN et al., 2002; DIAS et al., 2005).

Antes da aplicação dos testes de I-RM, todas as voluntárias passaram por duas sessões de familiarização com uma carga subjetiva e confortável em todos os exercícios que seriam utilizados nos testes, para a correção da execução dos movimentos.

Foram realizados os testes nos seguintes exercícios: flexão horizontal dos ombros e extensão dos cotovelos no supino reto com a barra, abdução dos ombros 
e extensão dos cotovelos no desenvolvimento com a barra, flexão dos cotovelos na rosca direta com a barra e extensão dos cotovelos no tríceps testa com a barra.

Para o cálculo da força relativa, utilizou-se a seguinte fórmula para os respectivos exercícios:

Força Relativa $=1-R M *(\mid /$ massa corporal $)$

\section{ANÁLISE ESTATIISTICA}

Os dados foram processados no SPSS 13.0, adotando o nível de significância de cinco por cento $(p<0,05)$. Foi verificada a normalidade dos dados (teste de Shapiro Wilk) e a homocedasticidade (teste de Levene), utilizando o teste t de Student para os dados paramétricos e o de Mann Whitney para os não paramétricos, além do teste t de Student com correção de Welch quando não verificada a homocedasticidade.

\section{RESULTADOS}

A Tabela I apresenta os valores antropométricos das voluntárias dos grupos treinadas (GT) e não treinadas (GNT), não havendo diferenças significativas entre os grupos.

Tabela I. Resultados das variáveis antropométricas dos grupos treinadas (GT) e não treinadas (GNT)

\begin{tabular}{lll}
\hline Variáveis & $\mathrm{GT}(\mathrm{n}=43)$ & $\mathrm{GNT}(\mathrm{n}=47)$ \\
\hline Massa Corp. $(\mathrm{kg})^{\mathrm{Mw}}$ & $57,91 \pm 6,36$ & $60,60 \pm 10,46$ \\
Mínimo & 45,40 & 45,30 \\
Máximo & 71,10 & 88,50 \\
Estatura $(\mathrm{m})^{\mathrm{t}}$ & $1,65 \pm 0,07$ & $1,64 \pm 0,05$ \\
Mínimo & 1,50 & 1,53 \\
Máximo & 1,80 & 1,78 \\
IMC & $21,25 \pm 1,58$ & $22,55 \pm 3,61$ \\
$\left(\mathrm{~kg} / \mathrm{m}^{2}\right)^{\mathrm{MW}}$ & 18,40 & 17,50 \\
Mínimo & 24,60 & 32,90 \\
Máximo &
\end{tabular}

Valores são média +desvio padrão. Massa Corp. = Massa Corporal; IMC = Índice de Massa Corporal; ${ }^{\text {MW }}=$ teste de Mann Whitney; ${ }^{\mathrm{t}}=$ teste $\mathrm{t}$ de Student. 
A Tabela 2 apresenta os resultados dos testes de I-RM absolutos e relativos à massa corporal respectivamente, indicando resultados significativamente maiores no GT em relação ao GNT em todos os testes realizados.

Tabela 2. Resultados dos testes de I-RM absolutos e relativos à massa corporal das voluntárias dos grupos treinadas (GT) e não treinadas (GNT)

\begin{tabular}{|c|c|c|c|c|}
\hline \multirow{2}{*}{$\begin{array}{l}\text { Variáveis } \\
(\mathrm{kg})\end{array}$} & \multicolumn{2}{|l|}{ Absoluto (kg) } & \multicolumn{2}{|l|}{ Relativo } \\
\hline & GT $(n=43)$ & GNT $(n=47)$ & $\mathrm{GT}(\mathrm{n}=43)$ & GNT $(n=47)$ \\
\hline Supino ${ }^{t}$ & $36,44 \pm 8,05 * *$ & $27,34 \pm 5,41$ & $0,63 \pm 0,13$ *** & $0,46 \pm 0,09$ \\
\hline Mínimo & 19,00 & 17,00 & 0,39 & 0,28 \\
\hline Máximo & 59,00 & 38,00 & 1,04 & 0,65 \\
\hline Desenv. MW & $27,93 \pm 4,57 * *$ & $22,70 \pm 4,10$ & $0,49 \pm 0,08 * *$ & $0,38 \pm 0,07$ \\
\hline Mínimo & 18,00 & 15,00 & 0,34 & 0,21 \\
\hline Máximo & 39,00 & 37,00 & 0,68 & 0,53 \\
\hline Rosca MW & $21,63 \pm 4,19 * *$ & $15,57 \pm 3,06$ & $0,37 \pm 0,07 * *$ & $0,26 \pm 0,06$ \\
\hline Mínimo & 12,00 & 10,00 & 0,21 & 0,13 \\
\hline Máximo & 32,00 & 24,00 & 0,56 & 0,43 \\
\hline Tríceps TestaMw & $18,09 \pm 5,24 * *$ & $12,55 \pm 2,57$ & $0,31 \pm 0,08 * *$ & $0,21 \pm 0,05$ \\
\hline Mínimo & 12,00 & 8,00 & 0,21 & 0,12 \\
\hline Máximo & 34,00 & 18,00 & 0,60 & 0,35 \\
\hline
\end{tabular}

Valores são média +desvio padrão. Supino = Flexão horizontal dos ombros e extensão dos cotovelos no Supino Reto com a Barra; Desenv. = Abdução dos ombros e extensão dos cotovelos no Desenvolvimento com a Barra; Rosca = Flexão dos cotovelos na Rosca Direta com a Barra; Tríceps Testa = Extensão dos Cotovelos no Tríceps Testa com a Barra. ${ }^{\mathrm{MW}}=$ teste de Mann Whitney; ${ }^{\mathrm{t}}=$ teste $\mathrm{t}$ de Student. ${ }^{*} *$ Indica diferença significante $(p<0,0 \mathrm{I})$.

A Tabela 3 apresenta os resultados da média de 70\% dos testes de I-RM dos quatro exercícios, em valores relativos à massa corporal, para referência de carga inicial em mulheres jovens treinadas e não treinadas.

Tabela 3. Valores de referência de carga inicial para a aplicação dos testes de I-RM em mulheres jovens treinadas e não treinadas (70\% |-RM)

\begin{tabular}{lll}
\hline Variáveis & Treinadas & Não Treinadas \\
\hline Supino & 0,44 & 0,32 \\
Desenv. & 0,34 & 0,27 \\
Rosca & 0,26 & 0,18 \\
Tríceps Testa & 0,22 & 0,15 \\
\hline
\end{tabular}

Supino = Flexão horizontal dos ombros e extensão dos cotovelos no Supino Reto com a Barra; Desenv. = Abdução dos ombros e extensão dos cotovelos no Desenvolvimento com a Barra; Rosca = Flexão dos cotovelos na Rosca Direta com a Barra; Tríceps Testa $=$ Extensão dos Cotovelos no Tríceps Testa com a Barra. 


\section{DISCUSSÃO}

Embora existam alguns fatores que dificultem a aplicação dos testes de I-RM, como a determinação da carga inicial e o tempo necessário para a realização do teste (BRENTANO et al., 2008) e a própria familiarização (DIAS et al., 2005), este ainda é um método muito utilizado para a avaliação da força muscular em vários estudos (LEVERITT et al., 2003; GLOWACKI et al., 2004; CAUZA et al., 2005; WESTPHAL; BAPTISTA; OLIVEIRA, 2006; MAYHEW et al., 2008; MCCURDY et al., 2008; SOUZA et al., 2008; CESAR et al., 2009; HUNGER et al., 2009), inclusive em diversas modalidades esportivas (KRAVITZ et al., 2003; BRECHUE; MAYHEW, 2009; DESGORCES et al., 20 I0; BRECHUE, MAYHEW, 20I2).

Os resultados apresentam que, mesmo diante da semelhança entre o GT e o GNT quanto às variáveis antropométricas, o GT obteve resultados nos testes de I-RM significativamente maiores, o que era esperado por serem praticantes regulares de treinamento com pesos. Neste caso, a experiência com o treinamento de força foi determinante para resultar em valores significativamente maiores nos testes de I-RM, o que não foi observado por Raso, Matsudo e Matsudo (2002), que não encontraram diferenças nos valores de I-RM no leg press $45^{\circ}$ entre experientes e inexperientes. Entretanto, o presente estudo avaliou mulheres jovens, enquanto que Raso, Matsudo e Matsudo (2002) avaliaram mulheres idosas.

McCurdy et al. (2008) realizaram um estudo em que foi feito o teste de I-RM no supino reto em sete mulheres jovens treinadas, obtendo resultado superior ao do presente estudo, porém a amostra apresentava características distintas, além do fato de ter sido utilizado somente o supino reto.

Westphal, Baptista e Oliveira (2006) estudaram sete mulheres treinadas, encontrando valores absolutos no teste de I-RM no supino reto próximos ao GT do presente estudo.

Mayhew et al. (2008) submeteram 103 mulheres jovens não treinadas ao treinamento de força, encontrando valores de I-RM no supino reto, absoluto e relativo à massa corporal, bem similares ao do presente estudo.

Dias et al. (2005b) realizaram um estudo com 15 mulheres não treinadas e encontraram valores de I-RM no supino reto e na rosca direta próximos aos do GNT do presente estudo no supino reto, embora superiores na rosca direta.

Materko, Neves e Santos (2007) estudaram 22 mulheres treinadas no teste de I-RM no desenvolvimento de ombros na máquina, com resultado menor que o valor encontrado no GT deste estudo, mas no presente estudo utilizou-se o desenvolvimento com a barra.

Como descrito acima, diversos estudos corroboram ou se aproximam muito aos resultados do presente estudo. Entretanto, nesta pesquisa foi realizada a aplicação 
de quatro diferentes exercícios para os membros superiores, e não somente um ou dois como acontece em trabalhos anteriores (DIAS et al., 2005b; WESTPHAL; BAPTISTA; OLIVEIRA, 2006; MATERKO; NEVES; SANTOS, 2007; MAYHEW et al., 2008; MCCURDY et al., 2008), além do fato de propor valores de referência para a carga inicial do teste de I-RM.

Tendo em vista que a força muscular é influenciada, dentre outros fatores, pela massa magra e área de secção transversa do músculo, é crescente a utilização de valores relativos dos testes de I-RM, levando também em consideração a massa corporal do avaliado, buscando uma avaliação mais apurada da força muscular (WESTPHAL; BAPTISTA; OLIVEIRA, 2006; BRENTANO et al., 2008; MAYHEW et al., 2008).

Deve-se ressaltar que este estudo priorizou a utilização de quatro exercícios livres, realizados com barras e anilhas, de modo que os resultados podem ser utilizados em qualquer academia de ginástica ou clube que tenha estes materiais.

Um fator limitante do estudo foi a não padronização da velocidade de execução dos movimentos e a realização de uma única sessão de I-RM para os respectivos exercícios, o que não diminui a importância da aplicação prática do estudo, visto que o objetivo do mesmo foi a proposição de valores de referência para a carga inicial de testes de I-RM em exercícios de membros superiores. Embora os resultados do presente estudo possam ser aplicados somente à população estudada, ou seja, mulheres jovens.

Outro fator limitante pode ter sido a realização dos testes de I-RM acontecerem em uma mesma sessão, o que poderia interferir no resultado da carga total dos respectivos exercícios. Entretanto, pensando no objetivo de proposição de carga inicial, isso parece não interferir na sua aplicação prática.

Este trabalho tem como aplicação prática fornecer valores de referência da carga inicial para a aplicação dos testes de I-RM em mulheres jovens treinadas e não treinadas em treinamento com pesos, sendo uma ferramenta para profissionais da educação física na otimização da avaliação da força. Sendo assim, sugere-se como carga inicial para o teste de I-RM, aproximadamente 70\% da média da carga máxima encontrada no presente estudo, para os diferentes exercícios, visto que esta é uma intensidade proposta como aquecimento específico no protocolo de I-RM (BROWN; WEIR, 200 I). A Tabela 3 pode ser utilizada para o cálculo da carga inicial, multiplicando-se a massa corporal da voluntária pelo valor da tabela para cada exercício em cada grupo. Por exemplo, em uma mulher de 50 kg não treinada, os valores de carga inicial dos testes de I-RM seriam: supino $16 \mathrm{~kg}$, desenvolvimento com a barra $13,5 \mathrm{~kg}$, rosca direta $9 \mathrm{~kg}$ e tríceps testa $7,5 \mathrm{~kg}$. 


\section{CONCLUSÕES}

Os resultados obtidos demonstraram que as mulheres jovens treinadas apresentaram maiores valores do que não treinadas nos quatro testes de I-RM realizados, de modo que a carga inicial para testes de I-RM deve ser diferente em mulheres treinadas e não treinadas. Este trabalho tem como aplicação prática servir como referencial para profissionais da área da educação física na orientação de cargas iniciais para a aplicação dos testes de I-RM em exercícios de membros superiores, em mulheres jovens treinadas e não treinadas.

\section{Load to the Application of I-RM Tests in Upper Limbs of Young Women Trained and Untrained}

ABSTRACT: The purpose of this study was to propose reference values to application of the I-RM tests in young women trained and untrained. 90 women, |8-3| years, grouped into: 43 Trained (GT) and 47 Untrained (GNT) were submitted to I-RM tests in the following exercises: bench press, shoulder press, biceps curl and lying triceps extension. GT showed higher values than GNT in absolute and relative terms: bench press $(\mathrm{GT}=36.44 \mathrm{~kg} / 0.63$ and $\mathrm{GNT}=27.34 \mathrm{~kg} / 0.46)$, shoulder press $(\mathrm{GT}=27.93 \mathrm{~kg} / 0.49$ and $\mathrm{GNT}=22.70 \mathrm{~kg} / 0.38)$, biceps curl (GT $=21.63 \mathrm{~kg} / 0.37$ and $\mathrm{GNT}=15.57 \mathrm{~kg} / 0.26)$ and lying triceps extension $(\mathrm{GT}=18.09 \mathrm{~kg} / 0.3 \mathrm{I}$ and $\mathrm{GNT}=12.55 \mathrm{~kg} / 0.2 \mathrm{I})$. The values found in this study can be used as references of load to application of I-RM tests.

KEY-WORDS: Physical education and training; physical assessment; women; muscle strength.

Cargo por el uso de las pruebas de I-RM en el miembro superior de las mujeres jóvenes entrenados y no entrenados

RESUMEN: El objetivo del estudio fue proponer puntos de referencia para la aplicación de las pruebas de IRM en mujeres jóvenes entrenados y no entrenados. 90 mujeres, | 8-3 | años, agrupados en: 43 entrenados (GT) y 47 no entrenados (GNT), se probaron para las pruebas de IRM en los siguientes ejercicios: prensa de banca, prensa de hombros, curl con barra y extensión de tríceps. GT mostró valores más altos en comparación con el GNT en términos absolutos y relativos: prensa de banca (GT $=36,44 \mathrm{~kg} / 0,63$ y GNT $=27,34 \mathrm{~kg} / 0,46)$, prensa de hombros (GT $=27,93 \mathrm{~kg} / 0,49$ y $\mathrm{GNT}=22,70 \mathrm{~kg} / 0,38)$, curl con barra (GT $=21,63 \mathrm{~kg} / 0,37$ y $\mathrm{GNT}=15,57 \mathrm{~kg} / 0,26)$ y extensión de tríceps $(\mathrm{GT}=18,09 \mathrm{~kg} / 0,31$ y GNT $=$ 12,55 kg/0,2 I). Los valores encontrados en el estudio pueden ser utilizados como referencia de cargo para la aplicación de las pruebas de I RM. PALABRAS CLAVE: Educación física y entrenamiento; evaluación física; mujeres; fuerza muscular. 


\section{REFERÊNCIAS}

BARBOSA, A. R. et al. Efeitos de um programa de treinamento contra resistência sobre a força muscular de mulheres idosas. Revista Brasileira de Atividade Física e Saúde, Londrina, v. 5, n. 3, p. 12-20, 2000.

BRECHUE, W. F.; MAYHEW, J. L. Upper-body work capacity and I rm prediction are unaltered by increasing muscular strength in college football players. Journal of Strength and Conditioning Research, Colorado Springs, v. 23, n. 9, p. 2477-2486, 2009.

BRECHUE, W. F; ; MAYHEW, J. L. Lower-body work capacity and one-repetition maximum squat prediction in college football players. Journal of Strength and Conditioning Research, Colorado Springs, v. 26, n. 2, p. 364-372, 2012.

BRENTANO, M. A. et al. Estimativa de força máxima em exercícios de musculação baseados em parâmetros antropométricos de homens e mulheres fisicamente ativos. Brazilian Journal of Biomotricity, Rio de Janeiro, v. 2, n. 4, p. 294-301, 2008.

BROWN, L. E.; WEIR, J. P. (ASEP) procedures recommendation I: accurate assessment of muscular strength and power. Journal of Exercise Physiology, v. 4, n. 3, p. I-21, 2001.

CAUZA, E. et al. The relative benefits of endurance and strength training on the metabolic factors and muscle function of people with type 2 diabetes mellitus. Archives of Physical Medicine and Rehabilitation, Philadelphia, v. 86, n. 8, p. I527-I 533, 2005.

CESAR, M. C. et al. The effect of local muscle endurance training on cardiorespiratory capacity in young women. Journal of Strength in Conditioning Research, Colorado Springs, v. 23, n. 6, p. 1637-1643, 2009.

DESGORCES, F. D. et al. Local muscular endurance and prediction of I repetition maximum for bench in 4 athletic populations. Journal of Strength and Conditioning Research, Colorado Springs, v. 24, n. 2, p. 394-400, 2010.

DIAS, R. M. R. et al. Influência do processo de familiarização para avaliação da força muscular em testes de I-RM. Revista Brasileira de Medicina do Esporte, São Paulo, v. I I, n. I, p. 34-38, 2005.

DIAS, R. M. R. et al. Impacto de oito semanas de treinamento com pesos sobre a força muscular de homens e mulheres. Revista Brasileira de Medicina do Esporte, São Paulo, v. I I, n. 4, p. 224-228, 2005b.

GLOWACKI, S. P. et al. Effects of resistance, endurance, and concurrent exercise outcomes in men. Medicine and Science in Sports and Exercise, Hagerstown, v. 36, n. 12, p. 21 1 9-2 1 27, 2004.

HUNGER, M. S. et al. Efeitos de diferentes doses de suplementação de creatina sobre a composição corporal e força máxima dinâmica. Revista da Educação Física, Maringá, v. 20, n. 2, p. 25I-258, 2009. 
HURLBUT, D. E. et al. Does age, sex or ACE genotype affect glucose and insulin responses to strength training? Journal of Applied Physiology, Bethesda, v. 92, n. 2, p. 643-650, 2002.

KRAEMER, W. J.; RATAMESS, N. A. Fundamentals of resistance training: progression and exercise prescription. Medicine and Science in Sports and Exercise, Hagerstown, v. 36, n. 4, p. 674-688, 2004.

KRAVITZ, L. et al. Prediction of I repetition maximum in high-school power lifters. Journal of Strength and Conditioning Research, Colorado Springs, v. 17, n. I, p. 167-172, feb. 2003.

LEVERITT, M. et al. Concurrent strength and endurance training: the influence of dependent variable selection. Journal of Strength and Conditioning Research, Colorado Springs, v. I7, n. 3, p. 503-508, 2003.

MATERKO, W.; NEVES, C. E. B.; SANTOS, E. L. Prediction model of a maximal repetition ( I - RM) based on male and female anthropometrical characteristics. Revista Brasileira de Medicina do Esporte, São Paulo, v. 13, n. I, p. 22-26, 2007.

MAYHEW, J. L. et al. Accuracy of prediction equations for determining one repetition maximum bench press in women before and after resistance training. Journal of Strength and Conditioning Research, Colorado Springs, v. 22, n. 5, p. 1570- 1577, 2008.

MAZZETTI, S. A. et al. The influence of direct supervision of resistance training on strength performance. Medicine and Science in Sports and Exercise, Hagerstown, v. 32, n. 6, p. I I 75-84, 2000.

MCCARTHY, J. P. et al. Compatibility of adaptive responses with combining strength and endurance training. Medicine and Science in Sports and Exercise, Hagerstown, v. 27, n. 3, p. 429-436, 1995.

MCCARTNEY, N. et al. The effects of strength training in patients with selected neuromuscular disorders. Medicine and Science in Sports and Exercise, Hagerstown, v. 20, n. 4, p. 362-368, 1988.

MCCARTNEY, N.; MCKELVIE, R. S. The role of resistance training in patients with cardiac diseases. Journal of Cardiovascular Risk, Ontario, v. 3, n. 2, p. 160-166, 1996.

MCCURDY, K. et al. The validity and reliability of the I-RM bench press using chain-loaded resistance. Journal of Strength and Conditioning Research, Colorado Springs, v. 22, n. 3, p. 678-683, 2008.

NAHAS, M. V. Atividade física, saúde e qualidade de vida: conceitos e sugestões para um estilo de vida ativo. Londrina: Midiograf, 200 I.

POEHLMAN, E. T. et al. Effects of endurance and resistance training on total daily energy expenditure in young women: a controlled randomized trial. Journal of Clinical Endocrinology and Metabolism, Baltimore, v. 87, n. 3, p. 1004-1009, 2002. 
RASO, V.; MATSUDO, S. M. M.; MATSUDO, V. K. R. A experiência de mulheres idosas em programas de exercícios com pesos não determina a performance no teste I-rm nem a resposta da percepção subjetiva de esforço. Revista Brasileira de Ciências do Esporte, São Paulo, v. 23, n. 3, p. $81-92,2002$.

RONTU, J. P. et al. One -repetition maximum bench press performance estimated with a new accelerometer method. Journal of Strength and Conditioning Research, Colorado Springs, v. 24 , n. 8 , p. $2018-2025,2010$

ROTH, S. M.; MARTEL, G. F.; IVEY, F. M. High volume, heavy-resistance strength training and muscle damage in young and older women. Journal of Applied Physiology, Bethesda, v. 88, n. 3, p. $1112-1118,2000$.

SOUZA, T. M. F. et al. Efeitos do treinamento de resistência de força com alto número de repetições no consumo máximo de oxigênio e limiar ventilatório de mulheres. Revista Brasileira de Medicina do Esporte, São Paulo, v. 14, n. 6, p. 513-517, 2008.

WESTPHAL, M.; BAPTISTA, R. R.; OLIVEIRA, A. R. Relações entre massa corporal total, massa corporal magra, área de seção transversa e I-RM em mulheres. Revista Brasileira de Cineantropometria \& Desempenho Humano, Florianópolis, v. 8, n. I , p. 52-57, 2006.

WILBER, C. A. et al. An epidemiological analysis of overuse injuries among recreational cyclists. International Journal of Sports Medicine, Stuttgart, v. 16, n. 3, p. 201-206, 1995.

Recebido em: 15 mar. 2012 Aprovado em: 08 jun. 2012

Endereço para correspondência: Thiago Mattos Frota de Souza Rua Maestro Mario de Tulio, 91 Bosque das Palmeiras Campinas-SP CEP: 13.086-705 